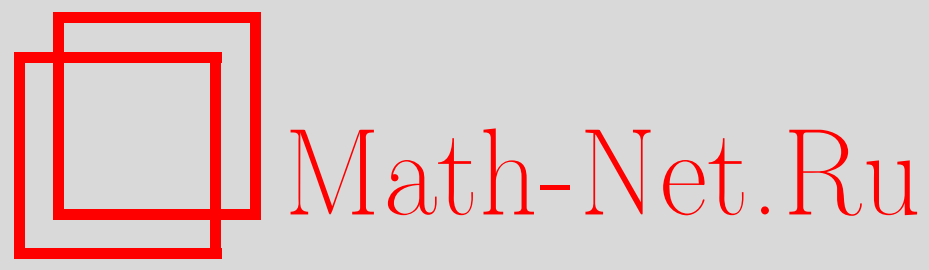

Б. Я. Казарновский, Экспоненциальные аналитические множества, Функи. анализ и его прил., 1997, том 31, выпуск 2, 15-26

DOI: https://doi.org/10.4213/faa456

Использование Общероссийского математического портала Math$\mathrm{Net.Ru}$ подразумевает, что вы прочитали и согласны с пользовательским соглашением

http://www . mathnet.ru/rus/agreement

Параметры загрузки:

IP : 54.92 .164 .108

26 апреля 2023 г., 12:37:30

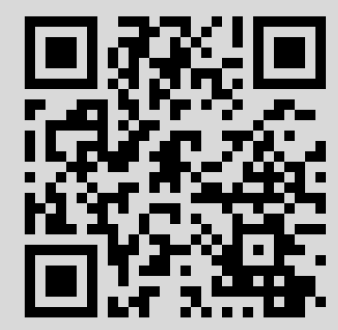


Функииональньй анализ и еәо приложения

1997, т. 31, вып. 2, с. 15-26

УдК 517.55

\title{
Экспоненциальные аналитические множества
}

\author{
(c) 1997. Б. Я. КАЗАРнОВСКИй
}

\section{§1. Введение}

1.1. Описание результатов. Экспоненциальные суммы (э.с.) - это функции в $\mathbb{C}^{n}$ вида $\sum_{\lambda \in \Lambda} c_{\lambda} \exp \langle z, \lambda\rangle$, где $c_{\lambda} \in \mathbb{C}$, а $\Lambda$ - конечное подмножество пространства показателей (т.е. сопряженного пространства $\mathbb{C}^{n *}$ ), называемое спектром э.с.; выпуклая оболочка спектра - это многогранник Ньютона э.с.

Экспоненциальное аналитическое множество (э.а.м.) - это множество совместных нулей конечной системы э.с. Если показатели экспонент системы вещественны и целочисленны (т.е. спектры принадлежат целочисленной решетке $\left.\operatorname{Re} \mathbb{C}^{n *}\right)$, то э.а.м. называется алгебраическим. Такое э.а.м. - прообраз алгебраического подмногообразия тора при отображении $\operatorname{exp:~} \mathbb{C}^{n} \rightarrow(\mathbb{C} \backslash 0)^{n}$. Квазиалгебраическим будем называть э.а.м., заданное уравнениями с вещественными показателями экспонент.

В п. 1.4 определена алгебраическая коразмерность э.а.м. Для алгебраических э.а.м. это коразмерность упомянутого подмногообразия тора. В общем случае она также равна коразмерности некоторого сопоставляемого системе алгебраического многообразия. Если это многообразие равноразмерно, то э.а.м. тоже называется равноразмерным. Кроме того, это сопоставление позволяет однозначно представить э.а.м. в виде объединения равноразмерных э.а.м. разных алгебраических коразмерностей.

Алгебраическая коразмерность э.а.м. не меньше его геометрической коразмерности (т.е. коразмерности как аналитического множества в $\mathbb{C}^{n}$ ). Геометрические компоненты э.а.м. коразмерности, меньшей алгебраической, назовем нетипичныли. Если э.а.м. задано одним уравнением или является алгебраическим, то обе коразмерности совпадают. Э.а.м., заданное системой двух э.с., не имеющих общего делителя в кольце э.с., равноразмерно и имеет алгебраическую коразмерность два. Пример неравенства коразмерностей дает система $\exp (\pi z)-1=\exp (z)-1=0$. Здесь алгебраическая и геометрическая коразмерности равны 2 и 1 , а нетипичная компонента - точка $z=0$.

В статье приведены некоторые результаты алгебраической теории размерности э.а.м., которую мы понимаем как априорное описание возможного множества нетипичных компонент э.а.м. данной алгебраической коразмерности. Пример доказываемое в п. 2.6

ПРЕДЛОЖЕНИЕ 1. Любая нетипичная компонента квазиалгебрачческого э.а.м. алгебраической коразмерности 2 являетсл аффинной гиперплоско-

\footnotetext{
^ Работа выполнена при поддержке грантов МНФ, AMS и РФФИ.
} 
стью. Множество таких аиперплоскостей имеет нулевую плотность и конечное количество оснований.

Основанием аффинного подпространства мы называем параллельное ему линейное подпространство в $\mathbb{C}^{n}$. Когда речь идет о квазиалгебраических э.а.м., то под термином аффинное подпространство мы понимаем подпространство, основание которого порождено своим пересечением с $\operatorname{Re} \mathbb{C}^{n}$. Проекции такого подпространства $L$ на пространства $\operatorname{Re} \mathbb{C}^{n}$ и $\operatorname{Im} \mathbb{C}^{n}$ имеют вещественные размерности, равные комплексной размерности пространства $L$, и называются его вещественной и мнимой частями. Мы говорим, что множество $m$-мерных подпространств такого типа имеет плотность $\mu$, если $\mu$ - плотность множества его мнимых частей. Плотностью множества $m$-мерных подпространств пространства $\mathbb{R}^{n}$ называется предел отношения количества подпространств, находящихся на расстоянии не более $r$ от нуля, $\mathrm{K} r^{n-m}$.

Приведем следствие предложения 1 в духе $[5,6]$.

СлЕдСТВИЕ 1. Если площадь объединения компонент коразмерности 1 множества нулей системь двух э.с. с вещественными показателями экспонент в шаре радиуса $R$ имеет порядок роста $R^{2 n-1}$, то эти э.с. имеют обиий делитель.

Так как площадь нулевой поверхности э.с. в шаре радиуса $R$ растет как $\rho R^{2 n-1}$, где $\rho$ — «периметр» ее многогранника Ньютона [7], то верно и обратное утверждение.

Отметим, что задача о нахождении общего делителя э.с. (см. п. 1.4) сводится к нахождению общего делителя полиномов. С другой стороны, из приведенных фактов вытекает, что делимость в кольце э.с. эквивалентна делимости в кольце целых функций $([3,4]$, в одномерном случае это старый результат Ритта [2]).

Опишем подробнее ситуацию в $\mathbb{C}^{1}$. Итак, если э.с. одного переменного взаимно просты, то множество мнимых частей их общих нулей имеет нулевую плотность. С другой стороны, такие э.с. могут иметь любое конечное количество общих нулей. Имеется давняя гипотеза, что «бесконечное количество обиих нулей приводит к существованию общего делителя в кольце э.с.». Если одна из э.с. есть $\exp (z)-1$, то это следует из результата, доказанного Сколемом в 40-х гг. в цикле работ по диофантовым уравнениям, который может быть сформулирован так: «Множество иелочисленных (m. е. принадлежаших $2 \pi \sqrt{-1} \mathbb{Z}$ ) нулей э.с. состоит из конечного (обычно пустого) множества точек и конечного (обычно нулевого) числа бесконечных в обе стороны арифметических прогрессий». Если приведенная гипотеза верна, то мы получаем законченную алгебраическую теорию размерности э.а.м. в $\mathbb{C}^{1}$, поскольку любое конечное множество точек в $\mathbb{C}^{1}$ можно представить как множество точек э.а.м. любой большей единищы алгебраической коразмерности. Кроме того, из утверждения гипотезы вытекает, что множество гиперплоскостей, являющихся нетипичными компонентами квазиалгебраического э.а.м. алгебраической коразмерности 2 , конечно.

Ситуация с делимостью и нулями э.с. в $\mathbb{C}^{1}$ сильно упрощается при введении зависимости от параметра. Э.с. с параметром - это э.с., коэффициенты которой являются полиномами от одномерного параметра. 
ТЕОРЕма 1. Если две э.с. с параметром имеют общий корень, меняющийся при изменении параметра, то они имеют общий делитель в кольце э.с. с параметром.

В утверждении теоремы э.с. могут быть заменены на полиномы от любого конечного набора алгебраически независимых голоморфных в области функций одного переменного.

При описании геометрии нетипичных компонент используется свойство квазиалгебраической экспоненциальной кривой (т.е. геометрически одномерной компоненты э.а.м.) иметь линейные асимптотики при уходе на бесконечность по вещественным частям. Это аналог свойства алгебраических кривых иметь степенные асимптотики при подходе к особенности. Вычисление возникающих рядов Пьюизо для плоских алгебраических кривых - классический источник возникновения диаграмм Ньютона [11]. Для экспоненциальных плоских кривых имеются аналогичные разложения в ряды Дирихле при подходе к бесконечности (см. п. 3.2).

В этой статье доказывается теорема о линейных асимптотиках только для плоских экспоненциальных кривых. В соответствии с этим и для достижения максимальной полноты формулировок мы ограничиваемся в основном случаем квазиалгебраических э.а.м. в $\mathbb{C}^{1}$ и $\mathbb{C}^{2}$. О переносе результатов на случай $n>2$ см. п. 1.3.

Вопрос о том, имеет ли произвольная (не квазиалгебраическая) экспоненциальная кривая на плоскости асимптотически линейный выход на бесконечность - это в действительности некоторый вопрос о геометрии расположения выпуклых многогранников в пространстве, снабженном комплексной структурой (см. конец п. 1.2).

Автор благодарит А. Г. Хованского за конструктивную критику результатов работы и их изложения.

\section{2. Формулировка результатов.}

ТЕоремА 2. Любая одномерная компонента плоского квазиалгебраического э.а.м. алгебраической коразмерности, большей единиць, является аффинной прямой.

Если любая плоская экспоненциальная кривая имеет асимптотически линейный выход на бесконечность, то ограничение квазиалгебраичности может быть снято.

ТЕОРема 3. Множество оснований аффинных подпространств, являющихся компонентами э.а.м., конечно.

ТЕОРемА 4. При $n \leqslant 2$ множество нетипичных компонент квазиалгебраического э.а.м. в $\mathbb{C}^{n}$ имеет нулевую плотность.

Из теоремы 4 вытекает, что при $n \leqslant 2$ справедлива

Теорема 5. Алгебраическая коразмерность равноразмерного э.а.м. в $\mathbb{C}^{n}$ равна $n$, если множество его изолированных точек не являетсл множеством нулевой плотности.

$\mathrm{C}$ другой стороны, множество корней общей системы $n$ э.с. имеет ненулевую плотность, вычисляющуюся по многогранникам Ньютона уравнений системы [7]. Если спектры э.с. вешественны, то плотность пропорциональна смешанному объ- 
ему многогранников Ньютона. При $n=1$ плотность равна $p / \pi$, где $p$ - длина отрезка Ньютона э.с.

Пусть $l, l^{+}$- одномерное комплексное подпространство в $\mathbb{C}^{n}$ и его полуплоскость с границей, принадлежащей $\operatorname{Im} \mathbb{C}^{n}$, a $L=z+l, L^{+}=z+l^{+}-$ аффинная прямая с основанием $l$ и ее полуплоскость.

ОПРЕДЕЛЕНИЕ 1. Пара $L, L^{+}$называется асимптотой квазиалгебраической экспоненииальной кривой, если для любого $\varepsilon$ при достаточно далеком от границы $t \in l^{+}$кривая пересекает $\varepsilon$-окрестность полуплоскости $t+L^{+}$по непустому множеству и пересекается с границей этой окрестности только вблизи границы полуплоскости $t+L^{+}$.

ТЕОРЕмА 6. Любая компонента плоской квазиалгебраической экспоненииальной кривой имеет асимптоту.

Легко показать, что множество оснований асимптот кривой конечно. Если кривая задается уравнением $f=0$, то асимптоты находятся из решений экспоненциальных уравнений одного переменного, написанных на сторонах многоугольника Ньютона. Соответственно плотность множества асимптот равна поделенному на $2 \pi$ периметру многоугольника Ньютона функции $f$. Вдали от плоскости мнимых частей кривая является возмущением множества своих асимптот, спадающим по мере удаления от этой плоскости. Возмущение каждой из асимптот разлагается в ряд Дирихле. Эта ситуация подробнее описана в 33 .

Приведем здесь один геометрический вопрос, связанный с линейными асимптотиками произвольных (не обязательно квазиалгебраических) плоских экспоненциальных кривых. Определение асимптоты произвольной экспоненциальной кривой отличается от приведенного выше тем, что $l$ может быть произвольной комплексной прямой (не обязательно порожденной своим пересечением с $\operatorname{Re} \mathbb{C}^{n}$ ), a $l^{+}$— произвольным не превосходящим $\pi$ углом на плоскости $l$ (не обязательно полуплоскостью).

Ребро выпуклого 4-мерного многогранника в $\mathbb{C}^{2}$ назовем крайним, если содержащая его аффинная комплексная прямая не пересекается с внутренностью многогранника. Существует ли многогранник без крайних ребер? Если такого многогранника не существует, то любая экспоненциальная кривая имеет асимптотически линейный выход на бесконечность. Наоборот, если $\Gamma \subset \mathbb{C}^{2}-$ такой многогранник, то (см. п. 3.3) кривая $f=0$, где $f$ - э.с. с многогранником Ньютона $\Gamma$, не имеет асимптотически линейного выхода на бесконечность.

1.3. Э.а.м. в многомерных пространствах. В действительности верно утверждение (размеры статьи не позволяют привести его вывода), что любая квазиалгебраическая экспоненииальная кривая имеет выход на бесконечность с линейной асимптотикой. Используя это, легко можно перенести вывод теоремы 2 в пространство произвольной размерности. При этом утверждение теоремы меняется следующим образом. Любая нетипичная компонента квазиалгебраического э.а.м. принадлежит иеликом некоторой аффинной гиперплоскости. Например, для системы трех э.с. от трех переменных, определяющей э.а.м. алгебраической коразмерности три, две из них могут вместе обратиться в нуль на некоторой гиперплоскости. Тогда э.а.м. содержит лежащую на этой гиперплоскости нетипичную кривую. 
Утверждение теоремы 5 остается верным для э.а.м. в многомерных пространствах без изменения формулировки.

1.4. Алгебраическая коразмерность. Комплексная $n$-мерная обмотка $N$-мерного тора — это гомоморфизм групп $\gamma: \mathbb{C}^{n} \rightarrow(\mathbb{C} \backslash 0)^{N}$, образ которого не содержится ни в каком подторе. Полином Лорана есть сумма характеров тора с постоянными коэффициентами. Для определения алгебраической коразмерности мы, следуя Г. Вейлю [1], будем рассматривать э.с. как ограничения полиномов Лорана на обмотке тора. Ясно, что прообраз любого алгебраического подмногообразия тора при отображении комплексной обмотки является э.а.м.

Пусть Г - конечно порожденная подгруппа пространства показателей (т.е. $\mathbb{C}^{n *}$ ), содержащая все спектры (показатели экспонент) исходной системы, $\mathbb{T}^{\Gamma}$ - тор еe характеров (гомоморфизмов в $\mathbb{C} \backslash 0), \gamma^{\Gamma}: \mathbb{C}^{n} \rightarrow \mathbb{T}^{\Gamma}$ - соответствующая комплексная обмотка, т.е. гомоморфизм, переводящий $z$ в характер $\chi(g)=\exp \langle z, g\rangle$. Тогда уравнения э.а.м. являются ограничениями на $\gamma^{\Gamma}\left(\mathbb{C}^{n}\right)$ однозначно определенных полиномов Лорана на $\mathbb{T}^{\Gamma}$. Совместные нули этих полиномов задают алгебраическое многообразие, которое мы обозначим через $M^{\Gamma}$.

ОПРЕДЕлЕнИЕ 2. Э.а.м. называется равноразмерныл, если равноразмерно (т.е. состоит из компонент одинаковой размерности) $M^{\Gamma}$. Коразмерность $M^{\Gamma}$ называется алгебраической коразмерностью э.а.м.

Как свойство равноразмерности, так и величина коразмерности многообразия $M^{\Gamma}$, не зависят от выбора подгруппы $\Gamma$. Действительно, если $\Omega, \Delta-$ подгруппы в Г, также содержащие спектры уравнений э.а.м., то $M^{\Gamma}-$ прообраз многообразий $M^{\Omega}, M^{\Delta}$ при отображениях проекции $\mathbb{T}^{\Gamma}$ на $\mathbb{T}^{\Omega}, \mathbb{T}^{\Delta}$, двойственных вложениям групп. Поэтому они все равноразмерны и их коразмерности одинаковы.

Каждое э.а.м. однозначно представляется как объединение конечного числа своих равноразмерных частей, имеющих разные алгебраические коразмерности. Это обстоятельство позволяет при выводе теорем считать э.а.м. равноразмерными, что мы и будем делать.

Для квазиалгебраических э.а.м. группа $\Gamma$, содержащая спектры системы, всегда предполагается выбранной в $\operatorname{Re} \mathbb{C}^{n *}$. Тогда ограничение $\gamma^{\Gamma}$ на Im $\mathbb{C}^{n}$ оказывается всюду плотной $n$-мерной обмоткой компактного подтора тора $\mathbb{T}^{\Gamma}$.

Из сказанного вытекают следующие свойства э.с. Кольцо э.с. 一 это кольцо полиномов Лорана бесконечномерного тора характеров дискретной аддитивной группы $\mathbb{C}^{n *}$. В кольце э.с. имеется наибольший общий делитель. Если спектры двух э.с. принадлежат некоторой конечно порожденной подгруппе группы $\mathbb{C}^{n *}$, то спектр их наибольшего общего делителя также принадлежит этой подгруппе (хотя, например, $\exp (z / m)+1$ делит $\exp (z)+1$ при любом целом $m$ ).

Следствием алгебраической независимости экспонент с целочисленно независимыми показателями является

Лемма 1. Если $U$ - открьтое подмножество в $\mathbb{C}^{n}$, то $\gamma^{\Gamma}(U)$ - всюду плотное в топологии Зариского подмножество в $\mathbb{T}^{\Gamma}$.

1.5. Запертые системы полиномиальных уравнений [12]. Здесь сформулированы утверждения о системах образующих идеала кольца полиномов Лорана, необходимые при выводе теоремы 5. 
Пусть $\mathbb{T}=(\mathbb{C} \backslash 0)^{N}$ - комплексный тор, $E=\operatorname{Re} \mathbb{C}^{N}$ и $E^{*}$ - сопряженное к $E$ пространство с двойственной целочисленной решеткой, точки которой мы отождествляем с характерами тора $\mathbb{T}$. Напомним, что конечное множество выпуклых многогранных целочисленных конусов в $E$ размерностей от нуля до $d$ называется $d$-мерным веером конусов [13], если любая грань конуса также является конусом этого веера, а пересечение любых двух конусов веера является их общей гранью.

Полином Лорана $f$ - линейная комбинация конечного множества характеров тора, взятых с ненулевыми коэффициентами. При $\varphi \in E$ определим $\varphi$-степень полинома как максимальное значение функционала $\varphi$ на этом множестве характеров. Старшую $\varphi$-однородную компоненту $f$ назовем $\varphi$-укорочением и обозначим через $f_{\varphi}$. Определим $\varphi$-укорочения системы $F=\left\{f^{1}, \ldots\right\}$, идеала $I$ и соответствующего алгебраического многообразия $M$, которое предполагается равноразмерным, как систему $F_{\varphi}=\left\{f_{\varphi}^{1}, \ldots\right\}$, идеал $I_{\varphi}$, порожденный полиномами $\left\{f_{\varphi}, f \in I\right\}$, и многообразие $M_{\varphi}$ нулей этого идеала. Положим $\Re_{M}=\left\{\varphi \in E: M_{\varphi} \neq \varnothing\right\}$.

Для любой системы $F$ определим множество $\Re_{F} \subset E$, состоящее из таких $\varphi$, что каждый из полиномов системы $F_{\varphi}$ содержит по крайней мере два монома. Рассмотрим в $\Re_{F}$ отношение эквивалентности $\varphi \sim \psi \Longleftrightarrow F_{\varphi}=F_{\psi}$. Ясно, что классы эквивалентности этого отношения являются конусами и определяют представление $\Re_{F}$ в виде объединения конусов некоторого веера.

ОПРЕДЕЛЕНИЕ 3. Конечную систему $F=\left\{f^{1}, \ldots, f^{q}\right\}$ образующих идеала $I$ назовем запертой, если

(1) при любом $\varphi \in E$ ее $\varphi$-укорочение $F_{\varphi}=\left\{f_{\varphi}^{1}, \ldots, f_{\varphi}^{q}\right\}$ является системой образующих идеала $I_{\varphi}$;

(2) при $\varphi \notin \Re_{M}$ среди $f_{\varphi}^{1}, \ldots, f_{\varphi}^{q}$ имеется характер с ненулевым коэффициенTOM.

ПРЕДЛОЖЕНИЕ 2. (1) В любом идеале кольиа полиномов Лорана имеется запертая система образующих. Если $F$ - запертая система образуюших идеала $I$, то $\Re_{M}=\Re_{F}$, а соответствуюший веер конусов $d$-мерен, где $d=\operatorname{dim} M$.

(2) Множество разных ч-укорочений многообразия $M$ конечно. Непустыле многообразия-укорочения равноразмерны и $d$-мерны.

СлЕДСТВИЕ 2. (1) Множество вещественных частей логарифмов точек многообразия $M$ расположено на ограниченном расстоянии от $\Re_{M}$.

(2) В дополнении $\kappa$ большому торическому шару с чентром в единиче расстояние от $M$ до $M_{\infty}$, где $M_{\infty}=\bigcup_{\varphi \neq 0} M_{\varphi}$, мало.

\section{§2. Вывод теорем 1-5}

2.1. Теорема 1. Если выбрать группу Г, содержащую спектры э.с., то получим алгебраическое многообразие $A \subset \mathbb{T}^{\Gamma} \oplus \mathbb{C}$, заданное двумя уравнениями.

Рассмотрим отображение проекции $A$ на $\mathbb{T}^{\Gamma}$. Из условия теоремы вытекает, что образ многообразия $A$ содержит некоторое открытое множество образа обмотки $\gamma^{\Gamma}$. Значит (лемма 1), этот образ содержит открытое по Зарискому множество. Поэтому коразмерность многообразия $A$ в $\mathbb{T}^{\Gamma} \oplus \mathbb{C}$ не превосходит единицы, что и требовалось доказать. 
2.2. Теорема 2. Пусть $K$ - компонента э.а.м. $X \subset \mathbb{C}^{2}$, отличная от аффинной прямой и координатная ось $\mathbb{C}_{z_{1}}$ с полуплоскостью $\operatorname{Re}\left(z_{1}\right)>0$ является ее асимптотой. Покажем, что алгебраическая коразмерность множества $X$ не превосходит единицы.

Выберем подгруппы $\Gamma_{1}, \Gamma_{2}$ в сопряженных координатным пространствах $\mathbb{C}_{z_{1}}^{*}, \mathbb{C}_{z_{2}}^{*}$ так, что группа $\Gamma=\Gamma_{1} \oplus \Gamma_{2}$ содержит спектры уравнений множества $X$. Тогда тор $\mathbb{T}^{\Gamma}$ с двумерной обмоткой $\gamma^{\Gamma}$ представляется в виде суммы торов $\mathbb{T}^{\Gamma_{1}} \oplus \mathbb{T}^{\Gamma_{2}}$ с одномерными обмотками. Рассмотрим проекцию $\pi: \mathbb{T}^{\Gamma} \rightarrow \mathbb{T}^{\Gamma_{2}}$ и при $t_{2} \in \mathbb{T}^{\Gamma_{2}}$ положим $X\left(t_{2}\right)=\pi^{-1}\left(t_{2}\right) \cap X^{\Gamma}$. Ограничение $\pi$ на обмотку $\gamma^{\Gamma}$ 一 это проекция пространства $\mathbb{C}^{2}$ на координатное пространство $\mathbb{C}_{z_{2}} ;$ поэтому (лемма 1) $\operatorname{codim} \pi\left(X^{\Gamma}\right)=0$. Отсюда следует, что $\operatorname{codim} X\left(t_{2}\right)=\operatorname{codim} X^{\Gamma}$ на открытом по Зарискому подмножестве $\mathbb{T}^{\Gamma_{2}}$. Пересечение дополнения этого подмножества с образом обмотки $\gamma^{\Gamma_{2}}$ является э.а.м. в $\mathbb{C}_{z_{2}}^{1}$. Таким образом, доказано

ПРЕДЛОЖЕНИЕ 3. Пусть $Y$ - э.а.м. в $\mathbb{C}^{2}$, содержащее одномерную компоненту, отличную от параллельного сдвига координатной оси $z_{2}=0$. Тогда если зафиксировать переменную $z_{2}$ в уравнениях, задаюших $X$, то алгебраическая коразмерность полученного э.а.м. в $\mathbb{C}_{z_{1}}$ может бить меньше алгебраической коразмерности э.а.м. $X$ только при $z_{2}$, принадлежащем некоторому собственному э.а.м. в $\mathbb{C}_{z_{2}}$.

Пусть $D \subset \mathbb{C}_{z_{2}}$ - круг малого радиуса с центром в нуле. Положим $\widehat{X}\left(z_{2}\right)=$ $X\left(\gamma^{\Gamma_{2}}\left(z_{2}\right)\right)$ и рассмотрим $\widehat{X}$ как семейство алгебраических подмногообразий $\mathbb{T}^{\Gamma_{1}}$ с экспоненщиальным параметром $z_{2} \in D$. Коразмерность слоя этого семейства постоянна при $z_{2} \neq 0$. Мы должны показать, что эта коразмерность не превосходит единицы.

Обмотка $\gamma^{\Gamma_{1}}$, ограниченная на прямую $\operatorname{Re} z_{1}=c$ в $\mathbb{C}_{z_{1}}$, при любом $c$ является всюду плотной обмоткой (см. п. 1.4) некоторого сдвинутого компактного подтора $\tau(c)$ тора $\mathbb{T}^{\Gamma_{1}}$. Если $c$ достаточно велико, то из определения 1 в п. 1.2 вытекает, что для любого числа $y$ найдется малое $\varphi(y) \in D$, такое, что точка в $\mathbb{C}^{2}$ с координатами $z_{1}=c+\sqrt{-1} y, z_{2}=\varphi(y)$ принадлежит $X$. Поэтому образ проекщии семейства $\widehat{X}$ на тор $\mathbb{T}^{\Gamma_{1}}$ содержит $\tau(c)$.

Для оценки коразмерности слоя мы сошлемся на приведенную ниже почти очевидную лемму 2. В качестве семейства аналитических множеств следует взять семейство $\widehat{X}$ в окрестности произвольной точки определенного выше сдвинутого компактного подтора $\tau(c)$. Если $\operatorname{codim} \widehat{X}(0)>0$, то ссылка на лемму 2 возможна. В противном случае (т. е. если исходное э.а.м. вместе с компонентой $K$ содержит и ее асимптоту) ссылка становится возможной после замены $\widehat{X}$ на замыкание множества $\widehat{X} \backslash \widehat{X}(0)$.

ЛЕмма 2. Пусть $D$ - круг на комплексной плоскости с иентром в нуле, $\{Y(c)\}$ - семейство аналитических множеств в окрестности нуля пространства $\mathbb{C}^{n}$, аналитически зависящих от параметра $c \in D, \operatorname{dim} Y(c)=d$ при $c \neq 0$, a codim $Y(0)>0$. Тогда если $\bigcup_{c \in D} Y(c)$ содержит окрестность нуля подпространства $\operatorname{Re} \mathbb{C}^{n}$, mо $d \geqslant n-1$.

2.3. Теорема 3. Пусть $f_{1}, \ldots, f_{k}$ - система уравнений, задающая э.а.м., компонентой которого является аффинное подпространство с основанием $E$. То- 
гда $E$ принадлежит гиперплоскости, лежащей на вещественной гиперплоскости из конечного набора гиперплоскостей, порожденных конусами, двойственными ребрам многогранников Ньютона э.с. $f_{i}$ (определение см. в п. 3.1). При ограничении э.с. системы на всевозможные сдвиги этих гиперплоскостей аналогичным образом получаем на них конечное число подпространств коразмерности два, так как для спектров ограничений э.с. на эти гиперплоскости имеется конечное число возможностей. Действуя подобным образом, мы получим содержащее $E$ конечное множество подпространств. Теорема доказана.

2.4. Теорема 4. Мы рассматриваем здесь случай нетипичных прямых на плоскости, так как теорема 5 доказывается отдельно. Ввиду теоремы 3 достаточно рассматривать множество параллельных нетипичных прямых. Предположим, что их основание - координатная прямая $z_{2}=0$, и рассмотрим семейство э.а.м. $X\left(z_{2}\right) \subset \mathbb{C}_{z_{1}}$, фиксируя $z_{2}$ в уравнениях исходного э.а.м. Как вытекает из конструкции п. 2.2, множество точек $z_{2}$, при которых алгебраическая коразмерность э.а.м. $X\left(z_{2}\right)$ равна нулю, образует э.а.м. в $\mathbb{C}_{z_{2}}$ алгебраической коразмерности, не меньшей, чем алгебраическая коразмерности исходного э.а.м. Поэтому по теореме 5 для случая э.а.м. в $\mathbb{C}^{1}$ оно имеет нулевую плотность. Теорема доказана.

2.5. Теорема 5. Пусть $\gamma$ есть $n$-мерная комплексная обмотка тора $\mathbb{T}$, ограничение которой на $\operatorname{Im} \mathbb{C}^{n}$ дает всюду плотную обмотку максимального компактного подтора $\tau$ тора $\mathbb{T}, M \subset \mathbb{T}-$ равноразмерное алгебраическое многообразие коразмерности, большей или равной $n+1$, исходное э.а.м. $X$ является $\gamma$-прообразом многообразия $M$ в $\mathbb{C}^{n}, X^{d}$ - множество дискретных точек в $X$ $\left(X^{d}=X\right.$ при $\left.n=1\right), X^{\operatorname{Im}}$ - проекция $X^{d}$ на $\operatorname{Im} \mathbb{C}^{n}$ и $B_{R} \subset \operatorname{Im} \mathbb{C}^{n}$ - шар радиуса $R$ с центром в нуле.

Мы построим непрерывную неотрицательную функцию $\varphi$ на $\tau$ со сколь угодно малым интегралом и такую, что при любом достаточно большом $R$ интеграл от нее по $\gamma\left(B_{R}\right)$ больше количества точек из $X^{\mathrm{Im}}$, содержащихся в этом шаре. В теории малочленов [8] доказывается следующая (довольно очевидная при $n=1$ )

Лемма 3. Если $B(y)$ - шар единичного радиуса в $\operatorname{Im} \mathbb{C}^{n}$ с чентром в $y$, то при любом $y$ количество связных компонент э.а.м. $X$ в области $B(y)+\operatorname{Re} \mathbb{C}^{n}$ не превосходит некоторого $\phi$.

Поэтому, когда функция $\varphi$ построена, для окончания доказательства остается сослаться на эргодическую теорему, т.е. утверждение о том, что среднее непрерывной функции по всюду плотной обмотке тора равно ее среднему по тору.

Положим $\widehat{\mathbb{T}}=\left\{t \gamma(x) \in \mathbb{T}: t \in \tau, x \in \operatorname{Re} \mathbb{C}^{n}\right\}$ и $\widehat{M}=M \cap \widehat{\mathbb{T}}$. Ясно, что $\widehat{\mathbb{T}}$ — подгруппа в $\mathbb{T}$, гомеоморфная $\tau \oplus \operatorname{Re} \mathbb{C}^{n}, \widehat{M}$ - вещественное аналитическое подмножество в $\widehat{\mathbb{T}}$, а $\gamma\left(\mathbb{C}^{n}\right) \subset \widehat{\mathbb{T}}, X=\gamma^{-1} \widehat{M}$. Обозначим через $\pi: \widehat{\mathbb{T}} \rightarrow \tau$ проектирование вдоль $\operatorname{Re} \mathbb{C}^{n}$.

Ниже будет показано, что при $n=1$ множество $\pi(\widehat{M})$ является аналитическим коразмерности, большей или равной двум. В этом случае нетрудно построить нужную функцию $\varphi$ на $\tau$. При $n=2$ структура множества $\pi(\widehat{M})$ может усложниться, но она все же такова, что нужная функция может быть построена. 
Из доказательства следующей леммы 4 видно, что $\pi(\widehat{M})$ остается аналитическим, если обмотка $\gamma$ не $(n+1)$-резонансна, т. е. если ее касательное пространство не содержит вектора с $n+1$ независимыми целочисленными соотношениями между координатами. В этом случае $X$ совпадает с множеством своих дискретных точек и расположено на конечном расстоянии от $\operatorname{Im} \mathbb{C}^{n}$, а доказательство теоремы проходит при любом $n$.

ЛЕмма 4. Случай $n=1: \pi(\widehat{M})$ - аналитическое множество коразмерности, большей или равной двум.

Случай $n=2: \pi(\widehat{M}) \subset \widehat{M}_{1} \cup D$, где $D$ - аналитическое множество, а $\widehat{M}_{1}$ - аналитическое множество в области $\tau \backslash D$. Их коразмерности больше двух. При любом $t \in \tau$ пересечение $D$ со сдвинутой обмоткой $t \gamma\left(\operatorname{Im} \mathbb{C}^{n}\right)$ состоит из дискретного множества аффинных прямых.

Покажем, как закончить доказательство теоремы 5, опираясь на лемму 4. Пусть $\tau_{1}$ - трансверсальный обмотке $\gamma\left(\operatorname{Im} \mathbb{C}^{n}\right)$ подтор тора $\tau$ коразмерности $n$ и $V_{\varepsilon}=\left\{t \in \tau: t=\gamma(z) t_{1}, z \in B_{\varepsilon}, t_{1} \in \tau_{1}\right\}$. Рассмотрим проекцию $\sigma: V_{\varepsilon} \rightarrow \tau_{1}$ вдоль обмотки $\gamma\left(\operatorname{Im} \mathbb{C}^{n}\right)$. Ее слоем в точке $t \in \tau_{1}$ является $t \gamma\left(B_{\varepsilon}\right)$ - шар радиуса $\varepsilon$, помещенный на сдвинутую обмотку. Из леммы 4 вытекает, что на $\tau_{1}$ существует непрерывная положительная функция $\psi$ со сколь угодно малым интегралом, равная единице в окрестности множества $\sigma\left(\pi(\widehat{M}) \cap V_{\varepsilon}\right)$. Отметим, что для существования такой функции требуется оценка коразмерности $\operatorname{codim} D>1$, т. е. более слабая, чем в утверждении леммы.

Продолжим функцию $\sigma^{*}(\psi) / \varepsilon^{n}$ до неотрицательной функции на $\tau$, равной нулю вне множества $V_{2 \varepsilon}$. Тор $\tau$ покрывается конечным числом сдвигов области $V_{\varepsilon}$. Построим для каждого из сдвигов функции указанного вида. Сумма этих функций, умноженная на $\phi$ из формулировки леммы 3 , и есть нужная нам Функция $\varphi$.

Переходим теперь к доказательству леммы 4. Здесь используются определения и факты, изложенные в п. 1.5.

При поднятии на алгебру Ли тора $\mathbb{T}$ вещественная часть обмотки становится $n$-мерным подпространством $\widehat{\gamma} \subset E$. Если $\widehat{\gamma}$ пересекает $\Re_{M}$ только в нуле, то, согласно следствию 2 из п. 1.5, проекция $\pi: \widehat{M} \rightarrow \tau$ является собственным отображением, и поэтому $\pi(\widehat{M})$ - аналитическое подмножество в $\tau$. Подобная ситуация имеет место для не $(n+1)$-резонансной обмотки и, в частности, при $n=1$. При $n=2$, если обмотка 3 -резонансна, $\widehat{\gamma}$ может пересекать $\Re_{M}$ по конечному множеству лучей $\varphi_{1}, \ldots, \varphi_{q}$.

Положим $D=\pi\left(\bigcup \widehat{M}_{\varphi_{i}}\right)$. Согласно следствию 2 , уходы на бесконечность и возникновение кривых в слое при проекции $\pi: \widehat{M} \rightarrow \tau$ могут происходить лишь над точками $\tau$, принадлежащими $D$. Поэтому $\widehat{M}_{1}$ - аналитическое подмножество в $\tau \backslash D$. Аналитичность $D$ вытекает из того, что слой отображения $\pi: \widehat{M}_{\varphi_{i}} \rightarrow \tau$ состоит из конечного числа параллельных $\varphi_{i}$ прямых, лежащих на конечном не зависящем от слоя расстоянии от начала координат $\operatorname{Re} \mathbb{C}^{2}$.

И последнее, о коразмерностях множеств $D$ и $\widehat{M}_{1}$. Снова рассмотрим трансверсальный обмотке $\gamma\left(\operatorname{Im} \mathbb{C}^{n}\right)$ подтор $\tau_{1} \subset \tau$ коразмерности $n$ и соответствующий комплексный подтор $\mathbb{T}_{1} \subset \mathbb{T}$. Пусть $m \in \widehat{M}$ и $t \in \tau$ таковы, что $\pi(m) \in t \tau_{1}$. Выберем малую окрестность $U$ точки $m$ и рассмотрим проекцию 
$\sigma: U \rightarrow t \mathbb{T}_{1}$ вдоль обмотки $\gamma$ (это комплексное продолжение рассмотренной выше проекции $\left.\sigma: V_{\varepsilon} \rightarrow \tau_{1}\right)$.

Если $m \in \widehat{M}_{1}$, то пересечение слоя $\sigma$ с $M$ дискретно, и, значит, $\sigma(M \cap U)$ задает росток $\mathscr{H}$ комплексного аналитического подмножества $\mathbb{T}_{1}$, причем $\operatorname{codim} \mathscr{H} \geqslant 1$. Тогда если $\mathscr{H}_{R}=\mathscr{H} \cap\left(t \tau_{1}\right)$, то также codim $\mathscr{H}_{R} \geqslant 1$. Отсюда, с учетом леммы 3 , вытекает нужная оценка коразмерности $\pi\left(\widehat{M}_{1}\right)$ в окрестности $\pi(m)$. Действительно, $\widehat{M}_{1} \cap U=\sigma^{-1} \mathscr{H}_{R} ;$ поэтому $\operatorname{codim} \widehat{M}_{1} \geqslant 2 n+1$, откуда $\operatorname{codim} \pi\left(\widehat{M}_{1}\right) \geqslant n+1$.

Теперь остается при $n=2$ показать, что $\operatorname{codim} \pi\left(\widehat{M}_{\psi}\right)>2$, если $\psi$ - одно из $\varphi_{i}$. Так как $\sigma$-слой $M_{\psi}$ является объединением конечного числа параллельных $\psi$ аффинных прямых (их пересечений с $U$ ), то $\sigma(M \cap U)$ задает росток комплексного аналитического множества $\mathscr{H} \subset \mathbb{T}_{1}$ коразмерности два или больше. Значит, $\operatorname{codim} \mathscr{H}_{R} \geqslant 2 ;$ следовательно, $\operatorname{codim} \sigma^{-1} \mathscr{H}_{R} \geqslant 4$ и $\operatorname{codim} \pi\left(\widehat{M}_{\psi}\right)>2$. Лемма 4 доказана.

2.6. Предложение 1. Из определения 2 следует, что пересечение э.а.м. с общим аффинным подпространством $\mathbb{C}^{n}$ является э.а.м. той же алгебраической коразмерности. Поэтому пересечение нетипичной гиперповерхности с общим $m$-мерным аффинным подпространством является нетипичной гиперповерхностью э.а.м. той же алгебраической коразмерности в $\mathbb{C}^{m}$. Отсюда получаем, что предложение 1 является следствием приведенных выше теорем об э.а.м. в $\mathbb{C}^{1}$ и $\mathbb{C}^{2}$.

\section{§3. Плоские экспоненциальные кривые}

3.1. Двойственные конусы. Пусть $Г$ - многогранник Ньютона э.с. $f$. Для каждой его грани $\Delta$ определим двойственный конус $K_{\Delta}$, состоящий из таких $z \in \mathbb{C}^{n}$, что линейная функция $\operatorname{Re}\langle z, \ldots\rangle$ в $\mathbb{C}^{n *}$ достигает максимума на многограннике $\Gamma$ во всех точках грани $\Delta$ и нигде больше. Конус $K_{\Delta}$ характеризуется тем, что на нем среди всех экспонент, входящих в $f$, максимальны и равны между собой по модулю те, показатели степени которых принадлежат $\Delta$.

Пространство $\mathbb{C}^{n}$ представляется в виде объединения попарно непересекаюшихся конусов $K_{\Delta}$. Обозначим через $\Re_{f}$ объединение конусов, двойственных граням ненулевой размерности. В конусах полной размерности, т. е. двойственных вершинам многогранника $\Gamma$, лидируют по величине модуля стояшие в этих вершинах мономы. При удалении от $\Re_{f}$ их лидерство усиливается; поэтому все нули э.с. $f$ располагаются в некоторой большой метрической окрестности $\Re_{f}$.

Если $f$ - э.с. с вещественными показателями, то имеем следующую картину разбиения $\mathbb{C}^{n}$ в объединение двойственных конусов. Вещественное пространство $\operatorname{Re} \mathbb{C}^{n}$ разбито в объединение конусов $H_{\Delta}$, двойственных граням многогранника $\Gamma$ из сопряженного пространства $\operatorname{Re} \mathbb{C}^{n *}$. Конус $K_{\Delta}$ имеет размерность, на $n$ большую размерности соответствующего $H_{\Delta}$, и равен $H_{\Delta}+\operatorname{Im} \mathbb{C}^{n}$.

3.2. Асимптоты квазиалгебраических плоских кривых. Приведем используемое ниже вспомогательное утверждение:

ЛЕмма 5. Замкнутая аналитическая кривая в $\mathbb{C}^{n}$ не может иеликом размещаться на конечном расстоянии от $\operatorname{Im} \mathbb{C}^{n}$. 
Для его доказательства достаточно заметить, что голоморфная функция $\left(C-z_{1}\right)^{-1} \cdots\left(C-z_{n}\right)^{-1}$ по принципу максимума не может достигать на этой кривой максимума модуля.

Если $n=2$, а $\Delta$ - сторона многоугольника Ньютона э.с. $f$, то $H_{\Delta}$ - полупрямая в $\operatorname{Re} \mathbb{C}^{2}$ и $K_{\Delta}$ - полуплоскость содержащей $H_{\Delta}$ комплексной прямой $l_{\Delta}$. Пусть $f_{\Delta}$ - часть $f$, написанная на стороне $\Delta$ многоугольника Ньютона $\left(f_{\Delta}\right.$ есть $\varphi$-укорочение э.с. $f$ при $\left.\varphi \in H_{\Delta}\right)$. Множество нулей функции $f_{\Delta}$ состоит из прямых с основанием $l_{\Delta}$.

Нетрудно убедиться, что часть множества нулей э.с. $f$, лежащая на конечном расстоянии от $K_{\Delta}$ и вдали от плоскости $\operatorname{Im} \mathbb{C}^{2}$, является возмущением множества нулей э.с. $f_{\Delta}$, затухающим по мере удаления от $\operatorname{Im} \mathbb{C}^{2}$. Отсюда вытекает, что аффинные прямые, на которых $f_{\Delta}$ обращается в нуль, являются асимптотами э.а.м. нулей э.с. $f$. Теперь, чтобы доказать теорему об асимптоте для плоских кривых, достаточно сослаться на лемму 5.

Возмущение асимптоты плоской квазиалгебраической кривой разлагается в ряд Дирихле. Точнее говоря, приведя э.с. к виду

$$
f=f_{0}(w)+\sum_{k=1}^{q} \exp \left(-\alpha_{k} z\right) f_{k}(w)
$$

где $0<\alpha_{1}<\cdots<\alpha_{q}, f_{0}, \ldots, f_{q}$ - э.с. от $w$ с вещественными показателями экспонент, а $f_{0}$ имеет при $w=0$ нуль кратности $k$, мы можем сформулировать

ПредлОЖЕНИЕ 4. Кривая $f=0$ в области $\{\operatorname{Re} z>\mathbb{C},|w|<\varepsilon\}$ является объединением либо совпадаюших, либо непересекающихся графиков функиий $w_{1}(z), \ldots, w_{k}(z)$, представимых рядами Дирихле вида $\sum c_{\lambda} \exp (-\lambda z) c \lambda$, пробегаюшим неограниченно возрастаюшую последовательность положительных чисел.

ДокАЗАтЕльство. Пусть $F(w)=w^{k}+a_{k-1} w^{k-1}+\cdots+a_{0}-$ полином Вейерштрасса для неявной функции $f(w, z)=0$ в области $\operatorname{Re} z>C$ при $w<\varepsilon$. Для получения этого полинома следует подставить переменные $X_{j}$ на место $\exp \left(-\alpha_{j} z\right)$, выписать полином Вейерштрасса полученной функции и произвести обратную подстановку. Коэффициенты полинома $F$ - сходящиеся ряды Дирихле со стремящимися к $-\infty$ показателями экспонент из полугруппы, порожденной $\left\{-\alpha_{j}\right\}$. Поэтому при $k=1$ получено разложение для $w$ нужного вида.

Ряды Дирихле со стремящимися к $-\infty$ последовательностями показателей экспонент (здесь положительные показатели допускаются), сходящиеся в областях вида $\operatorname{Re} z>\mathbb{C}$, образуют поле. В общем случае предложение 4 вытекает из алгебраической замкнутости этого поля. Для формальных рядов алгебраическая замкнутость доказана в [9]. Для сходящихся рядов (как и для формальных) можно доказать замкнутость и одновременно найти все решения методом диаграмм Ньютона ([11], детальный разбор см. в [14]). Единственное отличие от классического метода диаграмм состоит в том, что сопоставляемая моному $c \exp (-\alpha z) w^{m}$ точка плоскости $(\alpha, m)$ имеет не обе, а только одну целочисленную координату. Это отличие не препятствует прохождению классического алгоритма Ньютона.

3.3. Произвольные плоские кривые. Пусть теперь $f$ - э.с. двух переменных с комплексными показателями и многогранником Ньютона Г полной 
размерности. Нетрудно показать, используя лемму 5 , что любая компонента множества нулей заходит как угодно глубоко внутрь некоторой фиксированной большой метрической окрестности одного из конусов $K_{\Delta}$, где $\Delta$ - либо ребро, либо двумерная грань, плоскость которой является аффинной комплексной прямой.

В последнем случае любая аффинная комплексная прямая, являющаяся компонентой множества нулей э.с. $f_{\Delta}$, будет асимптотой множества нулей э.с. $f$.

Пусть $\Delta$ - ребро многогранника $\Gamma, R_{\Delta}$ - трехмерное вещественное подпространство в $\mathbb{C}^{2}$, содержащее конус $K_{\Delta}$, а $\pi$ - содержащаяся в $R_{\Delta}$ комплексная прямая. Множество нулей э.с. $f_{\Delta}$ представляет собой дискретное множество аффинных прямых с основанием $\pi$, расположенных на конечном расстоянии от $R_{\Delta}$. Поэтому, если плоскость $\pi$ пересекается с конусом $K_{\Delta}$, то на каждой из этих комплексных прямых имеется угол, на котором некоторое затухающее к его внутренности возмущение является частью множества нулей э.с. $f$, т. е. эти прямые - асимптоты. Наоборот, если плоскость $\pi$ пересекается с замыканием конуса $K_{\Delta}$ только в начале координат, то каждая компонента множества нулей э.с. $f_{\Delta}$ пересекает фиксированную окрестность конуса $K_{\Delta}$ по компактному множеству и поэтому не может иметь асимптоты с основанием $\pi$. Если ребро $\Delta$ не является «крайним» (см. п. 1.2), то возникает именно эта последняя ситуация. Поэтому если у многогранника $\Gamma$ нет крайних ребер, то кривая $f=0$ не имеет асимптоты.

\section{ЛиТЕРАТУРА}

1. Weil H. Mean Motion. Amer. J. Math., 60, 889-896 (1938).

2. Ritt G. F. Trans. Am. Math. Soc., 31, 680-686 (1929).

3. Гордон A. Я, Левин A. Я. Теорема о делении квазиполиномов. Функц. анализ и его прил., 5, вып. 1, 22-29 (1971).

4. Avanissian V., Gay R. Sur une transformation des fonctionelles analytiques et ses applications. Bull. Soc. Math. France, 103, №3, 341-384 (1975).

5. Shields A. On quotients of exponential polinomials. Pure Appl. Math., 16, No. 1, 27-31 (1963).

6. Ронкин А. Л. Теорема о делении квазиполиномов. Теория функций, функциональный анализ и его приложения, вып. 34, 1980, с. 104-111.

7. Казарновский Б. Я. О нулях экспоненциальных сумм. ДАН СССР, 257, вып. 4, 804-808 (1981).

8. Khovanskii A. G. Fewnomials. Am. Math. Soc., Transl. of Math. Monogr., Vol. 68.

9. MacLane S. Universality of formal power series fields. Bull. Am. Math. Soc., 45, No. 12, 888-890 (1939).

10. Арнольд В. И. Гюйгенс и Барроу, Ньютон и Гук. Наука, М., 1989.

11. Казарновский Б. Я. Укорочения систем уравнений, идеалов и многообразий (готовится к печати).

12. Kemph G., Knudsen F., Mamford D., Saint-Donat B. Toroidal embeddings, 1. Lect. Notes Math., Vol. 339, Springer-Verlag, 1973.

13. Брюно А. Д. Локальный метод нелинейного анализа дифференциальных уравнений. Наука, М., 1979. 